\title{
Teoria fondamentale degli spazii di curvatura costante.
}

\author{
(del prof. Eugenio Bettrant, a Bologna).
}

In una Memoria inserita nel t. VIl della prima seric di questi Annali (Roma 1866) ho cercato le superficie dotate della proprieta di arere le loro lince geodetiche rappresentate da equazioni lineari, ed ho trovato che questa proprietà si verifica per le sole supcrficie di curvatura costante e per certe variabili speciali che l'analisi del problema ha spontaneamente introdotte.

Nel presente scritto espongo i risultati molto più generali a cui mi ha condotto l'ulteriore evoluzione di quel concetto, coordinato ad alcuni prin-

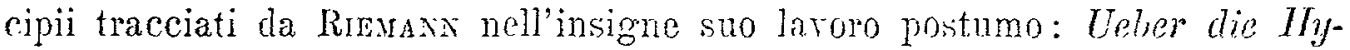
pothesen welche der Geometrie zu Grunde liegen, non ha guari pubrlicato dal sig. Denerixo nel XIII rolume delle Memorie di Gottinga. Spcro che le mie ricerche possano ajutare l'intelligonza di alcune parti di questo profondo lavoro.

Certe locuzioni di cui per amore di brevità faccio uso frequente non parranno, io credo, nè stenlate nè oscure a chi guardi più alla sostanza che alla forma. L'attento lettore non arrà da fare alcuno sforzo per intenderle senz'altra spiegazione, restandogli del resto piena facoltà di non attribuir loro che un significato meramente analitico.

L' espressione differenziale

$$
d s=R \frac{\sqrt{d x^{2}+d x_{1}^{2}+d x_{2}^{2}+\cdots+d x^{2}}}{x},
$$

dore $x, x_{1}, x_{2}, \ldots x_{n}$ sono $n+1$ variabili legate dall'equazione

$$
x^{2}+x_{1}^{2}+x_{2}^{2}+\cdots+x^{2}{ }_{n}=a^{2},
$$


mentre $R$ ed a sono due costanti, puo risguararsi come rapprescntante l'elemento lineare, ossia la distanza di duo punti infinitamente vicini, in uno spazio di $n$ dimensioni, ciascun punto del quale o definito da un sistema di valori delle $n$ coordinate $x_{1}, x_{2}, \ldots x_{i i}$. Ln forma di quell'espressione determina la natura di questo spazio.

Ponendo per brevità

$$
\Omega=V d x^{2}+d x_{1}^{2}+\cdots+c \cdot x^{2},
$$

le linee geodetiche dello spazio in quistione sono quelle che soddisfanno all' equazione

$$
\delta \int \frac{n}{x}=0
$$

colla condizione $x \delta x+x_{1} \delta x_{1}+\cdots+x_{i} \delta x_{n}=0$. Merce le solite trasformazioni della variazione dell'integrale, la prima equazione puo srilupparsi cosi:

$$
\int\left\{\delta x\left[\frac{\Omega}{x^{2}}+d\left(\frac{l x}{x \Omega}\right)\right]+\delta x_{1} \cdot d\left(\frac{d x_{1}}{x \Omega}\right)+\cdots+\delta x_{n} \cdot d\left(\frac{d x_{i}}{x \Omega}\right)\right\}=0,
$$

e, stante la relazionc che vincola le variazioni $\delta x, \delta x_{1}, \ldots \delta x_{n}$, dà luogo alle equazioni seguenti :

$$
\frac{\Omega}{x^{2}}+d\left(\frac{d x}{x ! n}\right)=k x, \quad d\left(\frac{d n_{1}}{x ! 2}\right)=k x_{1}, \cdots d\left(\frac{d x_{n}}{x \Omega}\right)=k x_{n},
$$

dove $k$ ì un fattore da determinare. Ora, moltiplicando queste cquazioni onilinatamente per $x, x_{1}, \ldots x_{1 b}$ e sommando, si ha

$$
d\left(\frac{x d x_{1}+x_{1} d x_{1}+\cdots+x_{n} d x_{n}}{x \Omega}\right)=h\left(x^{2}+x_{1}^{2}+\cdots+x_{n}^{2}\right)
$$

quindi, con riguardo alla (2), $k=0$; epperó

$$
\begin{gathered}
d\left(\frac{d x}{x \Omega}\right)+\frac{\Omega}{x^{2}}=0, \\
d x_{1}=c_{1} x \Omega, \quad d x_{2}=c_{2} x \Omega, \ldots, \quad d x_{n}=c_{n} x \Omega,
\end{gathered}
$$

dove $r_{1}, c_{2}, \ldots c_{i}$ sono costanti. (unesto ultimo $n$ equarioni, quadrate a sommate, lanno

$$
\Omega=-\frac{d x}{\sqrt{1-c^{2} \cdot x^{2}}},
$$


dove

$$
c=\sqrt{c_{1}^{2}+c_{2}^{2}+\cdots+c_{n}^{2}}
$$

Questo valore di: $\Omega$ rende identica la (3), della quale è percio inutile tener conto; mentre le $(4)$, colla climinazione di $x \Omega$ e susseguente integrazione, danno

$$
x_{1}=b_{1} x_{n 2}+b_{1}^{\prime}, \quad x_{2}=b_{2} x_{n}+l_{2}^{\prime} \ldots \quad x_{n-1}=b_{n-1} x_{n}+b_{n-1}^{\prime} .
$$

Dunque le linee geodetiche dello spazio considerato sono rappresentate da $n-1$ equazioni lineari fra le $n$ coordinate $x_{1}, x_{2}, \ldots x_{n}$, a simiglianza di ciò che ha luogo nel piano e nello spazio ordinario quando si fa uso di coordinate cartesiane, e nclle superficie di curvatura costante quando si fa uso delle variabili $u, v$ della citata Memoria. Fra i sistemi di linee geodetiche sono da notarsi specialmente quelli che si ottengono eguagliando tutte le coordinate, tranne una, ad altrettante costanti. Per ogni punto dello spazio passa una geodetica di ciascuno di questi sistemi, cui appartengono gli stessi assi coordinati delle $x_{1}$, delle $x_{2}, \ldots$, delle $x_{n}$, per ciascuno dei quali le restanti coordinate sono tutte nulle: conviene chiamarli sistemi delle $x_{1}$, delle $x_{2}, \ldots$ delle $x_{n}$.

ler ottenere la lunghezza dell'arco geodetico $\rho$ compreso fra due punti dati, si osservi che per la (5) si ha

$$
d \rho=I \frac{\Omega}{x}=-\frac{R \| x}{x \sqrt{1-c^{2} x^{2}}}
$$

donde

$$
c x=\frac{1}{\cosh \frac{p-a}{R}},
$$

$\rho_{0}$ essendo una costante arbitraria ed $x$ la funzione $\sqrt{a^{2}-x_{1}^{2}-x_{2}^{2}-\cdots-x_{n}^{2}}$. Indicando con $x_{1}^{0}, x_{2}^{0}, \ldots, x^{n}$ i valori delle coordinate al punto $\rho=0$, ciois all' origine dell'arco, e con $x^{0}$ il corrispondente valore della funzione s, si ha

$$
c x^{n}=\frac{1}{\cosh \frac{p_{n}}{n}}
$$


e quindi, eliminando $c$.

$$
x=\frac{x^{\prime \prime} \cosh \frac{\dot{s}_{0}}{R}}{\cosh \frac{\theta-\beta_{1}}{R}}
$$

equazione cui si può dare la forma

$$
\frac{x^{2} \operatorname{sen} h^{2} \frac{6}{h !}}{\cosh ^{2} \frac{p_{0}}{l l}}=2 x x^{0} \cosh \frac{?}{h} \cdots x^{2}-x^{4,2}
$$

D'altaa parte, irculosi dallo equazioni procedenti

$$
x \Omega=\frac{a^{2} l p}{h}=\frac{1}{c^{2}} a \operatorname{tg} h \frac{p-i_{0}}{R}
$$

le (4) danno

$$
x_{1}=a_{1}+\frac{c_{1}}{c^{2}} \operatorname{tg} \cdot h_{0} \frac{\hat{p}-p_{0}}{R}, \quad x_{2}=a_{2}+\frac{c_{3}}{c^{2}} \operatorname{tg} \cdot h_{0} \frac{p-p_{1}}{h}, \quad \text { ccc. }
$$

ovvero, sostituendo alle costanti $a_{1}, a_{2}, \ldots$ lo $x_{1}^{\prime \prime}, x_{2}^{\prime \prime}, \ldots$,

$$
x_{1}-x_{1}^{n}=c_{1} x x^{0} \operatorname{senh} \frac{\rho}{l}, \quad x_{2}-x_{2}^{n}=c_{2} x^{2} x^{0} \operatorname{senh} \frac{p}{h}, \quad \text { cec. }
$$

donde, quadrando e sommando,

$$
2\left(u^{2}-x_{1} x_{1}^{0}-x_{2} x_{2}^{0}-\cdots-x_{n} x_{n}^{0}\right)-x^{2}-x^{u^{2}}=c^{2} x^{2} x^{0^{2}} \operatorname{senh}{ }^{2}-\frac{?}{h} .
$$

Quest' equazione, in virta dolle (6) (7), dia finalmente

$$
\cosh \frac{\hat{i}}{R}=\frac{a^{2}-x_{1} x_{1}^{n}-x_{2} x_{2}^{0}-\cdots-x_{n} x_{n}^{0}}{\sqrt{\left(a^{2}-x_{1}^{2}-x_{2}^{2}-\cdots-x_{n}^{2}\right)\left(a^{2}-x_{1}^{n^{3}}-x_{2}^{n^{2}}-\cdots-x_{n}^{0}{ }^{2}\right)}},
$$

e questa c la formola generale clie porge la lunghezza di un arco geodetico in funzione delle coordinate dei snoi termini.

Supposte reali le variabili $x, x_{1}, \ldots x_{n}$ e le costanti $P$, $a$, il limite dello spazio di $n$ dimensioni qui considcrato è lo spazio di $n-1$ dimensioni rato dall' equazione

$$
x_{1}^{2}+x_{2}^{2}+\cdots+x^{2}{ }_{n}=a^{2} .
$$


Dentro questo limite, cios̀ per

$$
x_{1}^{2}+x_{2}^{2}+\cdots+x_{n}^{2}<a^{2}
$$

Il primo spazio è continuo e semplicemente connesso. Dalla (8) risulta poi che i punti appartenenti allo spazio limite sono tutti a distanza infinita.

Due elementi lincari $d s$, is uscenti da uno stesso punto $\left(x_{1}, x_{2}, \ldots x_{n}\right)$ e producenti rispettiramente le variazioni $d x_{1}, d x_{2}, \ldots d x_{n}$ e $\delta x_{1}, \delta x_{2}, \ldots \delta x_{n}$, sono fra loro ortogonali quando soddisfanno la relazione

$$
d x \delta x+d x_{1} \delta_{x_{1}}+\cdots+t x_{12} \delta x_{n}=0
$$

Consideriamo per esempio lo spazio di $n-1$ dimensioni $x_{1}=0$ e supponiamo che da un punto di esso escano due elementi lineari, l'uno ds esistente nello spazio stesso, l'altro $\delta s$ diretto secondo la geodetica dol sistema $x_{1}$ passante pei questo punto. In tal caso si ha

$$
x_{1}=0, d x_{1}=0, \delta x_{2}=\delta x_{3}=\cdots=\delta x_{n}=0, \delta x=0
$$

cpperỏ la condizione di ortogonalità $\dot{a}$ soddisfatta: vale a dire che ciascuna geodetica del sistema $x_{1}\left(0\right.$ più in generale $x_{r}$ ) è ortogonale allo spazio $x_{1}=0$ (risp. $x_{r}=0$ ) nel punto in cui lo incontra. In particolare dunque all'origine delle coordinate le dirczioni degli $n$ assi sono tutte ortogonali fra loro. Si dimostra con eguale facilità che l'asse $x_{v}$ o ortogonale a tutti gli spazii $x_{r}=$ cost. Le $n$ geodetirhe condotte da un punto arbitrario dello spazio nei sistemi $x_{1}, x_{2}, \ldots x_{n}$ riescono perpendicolari agli spazi di $n-1$ dimensione $x_{1}=0, x_{2}=0, \ldots x_{j i}=0$, analogamente a quel che ha luogo nel piano e nell' ordinario spazio quando si usano coordinate rettangole. Chiamando $X_{1}, X_{a}, \ldots X_{a}$ le porzioni di queste geodetiche comprese fra il punto dato e gli spazii cui sono rispettivamente perpendicolari, si ha

$$
X_{r}=\frac{n}{2} \operatorname{lng} \frac{\sqrt{x^{2}+x_{r}^{2}}+x_{r}}{\sqrt{x^{2}+x_{r}^{2}}-x_{r}} .
$$

Consideriamo il completo sistema relle geodetiche usconti dal punto doterminato $\left(x_{1}^{0}, a_{2}^{n}, \ldots x_{n}^{0}\right)$. T:so is rappresentato dal seguente sistema d'equazion: differenziali, l'ultima delle quali ì una conseguenza delle prime,

$$
\frac{d x_{1}}{x_{1}-x_{1}^{0}}=\frac{d x_{2}}{x_{2}-x_{2}^{0}}=\cdots=\frac{d x_{n}}{x_{n}-x_{n}^{0}}=\frac{d x}{x-\frac{z}{x}},
$$


dove si c̀ posto per brevità

$$
z=a^{2}-x_{1} x_{1}^{n}-x_{2} x_{2}^{0}-\cdots-x_{n} x_{n}^{0} .
$$

La condizione (10) fornisce come equazione differenziale dello spazio di $n-1$ dimensioni ortogonale a tutte queste geodetiche la seguente

$$
\frac{d z}{z}=\frac{d x}{x},
$$

donde integrando

$$
\frac{a^{2}-x_{1} x_{1}^{\prime \prime}-x_{2} x_{2}^{0}-\cdots-x_{n} x_{n}^{0}}{\sqrt{a^{2}-x_{1}^{2}-x_{2}^{2}-\cdots-x_{n}^{2}}}=C .
$$

Confrontando questa equazione colla (8) si vede che lo spazio definito da essa è anche il luogo dei punti equidistanti dal punto $\left(x_{1}^{0}, x_{2}^{0}, \ldots x^{0}{ }_{n}\right)$, c cliamando $\rho$ la distanza costante si ha

$$
C=\sqrt{a^{2}-x_{1}^{0^{2}}-x_{2}^{\prime l^{3}}-\cdots-x^{0}{ }_{n}^{2}} \cdot \cosh \frac{P}{h}=x^{0} \cosh \frac{P}{R} .
$$

Siccome l'equazione (12), pel modo in cui fu ottenuta, sussiste anche quando il punto $\left(x_{1}^{0}, x_{2}^{0}, \ldots x^{0}{ }_{n}\right)$ va all' infinito, cioè quando $x^{0}$ diventa nullo e $\rho$ infinito, cosi si vede che in questo caso il prodotto $x^{0} \cosh \frac{\rho}{l} \operatorname{con}-$ verge verso un limite finito, che non può differire da quello del prodotto $\frac{1}{2} x^{0} e^{\frac{\ell}{l}}$. Quindi scrivendo $\rho^{\prime}-\rho$ invece di $\rho$ o facendo andare all'infinito il punto $\left(x_{1}^{0}, x_{2}^{0}, \ldots x^{0}{ }_{n}\right)$, mentre $\rho$ rimane costante, si ottiene al limite l'equazione

$$
\frac{a^{2}-x_{1} x_{1}^{4}-x_{2} x_{2}^{0}-\cdots-x_{11} x^{11}}{\sqrt{a^{2}-x_{1}^{6}-x_{2}^{2}-\cdots-x^{4}}}=16 e^{-\frac{e}{\pi}},
$$

dove

$$
x_{1}^{0^{2}}+x_{2}^{3^{2}}+\cdots+x_{n}^{0^{2}}==a^{2},
$$

e quest'equazione rappresenta un sistema di spazii ad $n-1$ dimensioni, che possono essere definiti come le trajettorie ortogonali di tutte le geodetiche convergenti verso uno stesso punto all'infinito $\left(x_{1}^{0}, x_{2}^{n}, \ldots x_{n}^{0}\right)$. Le varie trajettorie sono distinte fra loro dai valori del parametro $\rho$, che esprime la distanza costante fra una qualunque di esse e la trajettoria determinata $\rho=0$. La costante $k$ ̀̀ data quando è dato uu punto di quest'ultima trajettoria. 
Ora si dimostrera che la natura dello spazio fin qui considerato e tale che, limitandone una porzione qualinque e trasportandola in una posizione diversa da quella che prima occupava, si puó sempre ottenerne la sovrapposizione con un'altra porzione corrispondente dello stesso spazio. Per concepire come ció possa accadere si immagini disseminato in quella porzione di spazio un numero $x^{n}$ di punti, infinitamente vicini tra loro, e riuniti a due a due dagli archetti geodetici che no misurano le mutue distanze. Ció posto, la sorrapponibilità della qualo si tratta consiste in questo, che in ogni altra parte dello spazio considerato si possono disseminare dei punti, ad esso appartenenti, i quali lanno fra loro le medesime distanze mutue e la medesima disposizione che avevano quelli della porzione immaginata; dimodochè il reticolo $n^{\text {picte }}$ formato dalle linee congiungenti i punti contigui di quosta può esscre completamente identificato col reticolo analogro dell'altra porziono, senza che i legami di essi derano essero in alcun punto rutti o duplicati. Le alterazioni che il primo reticolo dere subire per identificarsi col secondo non possono del resto riescire apparenti che quando si considerano l'uno e l'altro in rapporto ad uno spazio avente più di n dimensioni: finchè cio non accarle i duo reticoli presentano il carattcre delleguaglianza por congruenza o per simmetria. Quest'ul-

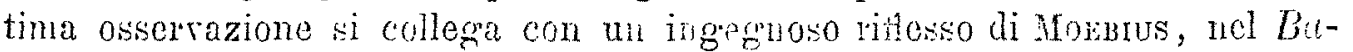
rycontrische Culcul, p. Is!.

Suppongasi dappima riferito lo spazio an un nuoro sistoma di assi goodetici dello $y_{1}, y_{2}, \ldots y_{n}$, arenti la stussa origine dei primi el ortogonali frit loro al pari di questi. Siccome tutte le linee goodctiche sono rappresontate da equazioni lineari, cosi è chiaro che le sostituzioni per passare dalle variabili $x$ alle rariabili y derono essere lincari: ma è facile convincersi inoltre che la loro forma dev'esscr quella denominata ortogronale. Infatti la forma (8) mostra clie la distanza dall'origine ad un junto qualunque $\left(x_{1}, x_{2}, \ldots x_{n}\right)$ dipende solamente dalla funzione $x_{1}^{2}+x_{2}^{2}+\cdots+x^{2}{ }_{n}$. Si avia dunque

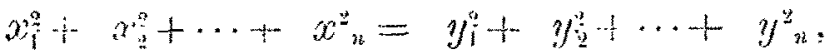

$$
\begin{aligned}
& d d_{1}^{2}+d x_{2}^{2}+\cdots+d x_{n}^{2}=d y_{1}^{2}+d y_{2}^{2}-\cdots+d y_{n}^{2},
\end{aligned}
$$

epperò

$$
\frac{d x^{2}+d x_{1}^{2}+\cdots+d y^{2}}{x^{2}}=\frac{d y^{2}+d y_{1}^{2}+\cdots+d y^{2}}{y^{2}}
$$


Questa identita di forma dei dne elementi rende manifesto che dur peticoli in cui i vertici corrispondenti fossero legnti dalle equazioni

$$
x_{1}=y_{1}, \quad r_{2}=y_{2}, \cdots \quad x_{n}=y_{i n}
$$

sarebbero perfettamente sovrapponibili. Ora è chinro che il secondo di questi reticoli non sarebbe altro che il primo girato intorno all' origine insicme coi primitivi assi, fino a che questi prendessero lo lihezioni dei nnovi. E dunque provato che la sovrapponibilità di cui si parlava ha effettivamente luogo quando lo spostamento si riduce ad una semplice rotazione intomo all origine. Anzi, siccome si potrebbe porre più generalmente

$$
x_{1}= \pm y_{1}, \quad x_{2}= \pm y_{2}, \ldots \quad x_{n}=\ldots y_{n}
$$

con lihertà di combinare $\mathrm{i}$ segni in modo qualunque, cosi è chiaro che oltre l'egnaglianza pu congruenza ri sono più specic degraglianza per simmetria.

Ioichè un cambiamento d'assi, restaudo fissa l'origine, non muta la forma dell'elemento lineare, resta ora a cercare l'effetto di un cambiamento d'origine. F poichè, preso nello spazio un punto qualunque, si può gia supporre diret to verso di esso l'asse delle $x_{1}$, cosi è lecito prendero la nuova origine su questo stesso asice, nel punto $x_{1}=a_{1}$. La nuova transformazione da eseguire consiste dunque nel mantenere l'asse delle $x_{1}$ ed i prececienti sistemi coordinati delle $x_{2}, x_{3}, \ldots x_{n}$, e nel sostituire al sistema delle geodetiche perpendicolari allo spazio $x_{1}=0$ quello delle geodetiche perpondicolari allo spazio $x_{1}=a_{1}$ fra le quali si trova il primitivo asse delle $x_{1}$. Le nuove coordinate si chiameramo $y_{1}, y_{2}, \ldots y_{i 2}$ e si chiamera $b$ una costante avente, rispetto a queste, lo stesso ufficio della costante a rispetto alle $x$. Cosi si denomineranun $Y_{1}, Y_{2}^{\gamma}, \ldots Y_{n}$ le geodetiche analoghe allo $X_{1}, X_{2}, \ldots X_{n}$ e si avrà manifestamente, come molla (11),

$$
Y_{r}=\frac{R}{2} \log \frac{\sqrt{y^{2}+y^{2}}+y_{r}}{\sqrt{y^{2}+y^{2}}-y_{r}} .
$$

Ciò posto, si osscrvi che, rimanendo invaruati i primitiri sistemi delle $x_{2}, x_{3}, \ldots x_{n}$. si la dapprima, per essi, $\lambda_{r}=Y_{r}$ o quinsli

$$
\frac{x}{x}= \pm \frac{y}{y} \quad \text { yer } \quad r=2,3, \ldots n \text {. }
$$

Quarkando e sommamio prima queste oquazioni, poi le Joro nifferenziali, si 
hanno le due formole

$$
\begin{aligned}
\left(u^{2}-x_{1}^{2}\right) y^{2} & =\left(b^{2}-y_{1}^{2}\right) x^{2}, \\
\frac{a^{2}}{x^{2}}+d\left(\frac{a}{x}\right)^{2}-d\left(\frac{x_{1}}{x}\right)^{2} & =\frac{a^{2}}{y^{2}}+d\left(\frac{b}{y}\right)^{2}-d\left(\frac{y_{1}}{y}\right)^{2},
\end{aligned}
$$

dove $\Theta^{2}=d y^{2}+d y_{1}^{2}+\cdots+d y^{2}$. In secondo luogo se si consideranc, sull'asse delle $x_{1}$, le porzioni $X_{1}^{0}, Y_{1}^{0}$ intercette fra lo due origini 0 il punto in cui l'asse stesso $\dot{i}$ intersecato dallo spazio $x_{1}=x_{1}$, si ha

$$
X_{1}^{0}=\frac{n}{2} \log \frac{a+x_{1}}{a-x_{1}}, \quad Y_{1}^{+}=\frac{n}{2} \log \frac{b+y_{1}}{b-y_{1}},
$$

mentre la distanza delle lue origini è data da

$$
\frac{R}{2} \log \frac{a+a_{1}}{a-a_{1}}
$$

È chiaro dunque che bisogna porre

$$
X_{1}^{a}=Y_{1}^{u}+\frac{n}{2} \log \frac{a+n_{1}}{a-u_{1}}
$$

$\operatorname{cioè~}$

$$
\frac{\left(a+r_{1}\right)\left(a-a_{1}\right)}{\left(a-x_{1}\right)\left(a+a_{1}\right)}=\frac{b+y_{1}}{b-y_{1}}
$$

donde

$$
y_{1}=\frac{a b\left(x_{1}-a_{1}\right)}{a^{2}-a_{1} x_{1}}, \quad x_{1}=\frac{a\left(a y_{1}-1-a_{1} b\right)}{a b+1 \cdot a_{1} y_{1}} .
$$

Queste due formole danno lnogo alle relazioni

$$
a^{2}-x_{1}^{2}=\frac{a^{2}\left(a^{2}-a_{1}^{2}\right)\left(b^{2}-y_{1}^{2}\right)}{\left(a b+a_{1} y_{1}\right)^{2}}, \quad b^{2}-y_{1}^{2}=\frac{b^{2}\left(a^{2}-a_{1}^{2}\right)\left(a^{2}-x_{1}^{2}\right)}{\left(a^{2}-a_{1} x_{1}\right)^{2}},
$$

le quali, combinate opportunamente colla prima delle (15), conducono a queste altre due:

$$
\begin{aligned}
& \frac{a}{a^{2}} \sqrt{a^{2}-a_{1}^{2}}=a_{1} \frac{b}{y}+a_{1} \frac{y_{1}}{y}, \\
& \frac{x_{1}}{a^{\prime}} \sqrt{a^{2}-a_{1}^{2}}=a_{1} \frac{b}{y}+a \frac{y_{1}}{y}
\end{aligned}
$$


donde

$$
d\left(\frac{a}{x}\right)^{2}-d\left(\frac{x_{1}}{x}\right)^{2}=d\left(\frac{b}{y}\right)^{2}-d\left(\frac{y_{1}}{y}\right)^{2}
$$

In virtù di quest'ultima equazione, la seconda delle (15) dà

$$
\frac{d x^{2}+d x_{1}^{2}+\cdots+d x^{2}{ }^{2}}{x^{2}}=\frac{d y^{2}+d y_{1}^{2}+\cdots+d y^{2}}{y^{2}}
$$

donde consegue che l'espressione dell'elemento lineare conserva la stessa forma anche mutando l'origine, e quindi, per un ragionamento analogo a quello di pocanzi, che la sovrapponibilità ha luogo in ogni caso, poichè basterebbe ora far uso di una nuova sostituzione ortogonale per rendere $i$ nuovi assi affatto indipendenti dai primi.

Le $(14)\left(15,1^{a}\right)(17)$ danno

$$
x_{r}= \pm \frac{a y_{r} \cdot \sqrt{a^{2}-a_{1}^{2}}}{a b+a_{1} y_{1}} \quad \text { per } r=2,3, \ldots n
$$

da cui e dalla $\left(16,2^{a}\right)$ si conclude che la più generale trasformazione d'assi ha luogo per mezzo di sostituzioni omografiche.

Prescindendo da questa trasformaziono delle coordinate $x_{1}, x_{2}, \ldots x_{n}$ in altre della stessa specie, vi sono altre trasformazioni che danno all' elemento una forma notabile. Quella che si potrebbe chiamar polare si ottiene ponendo primieramente

$$
x_{1}=r \lambda_{1}, \quad x_{2}=r m_{2}, \ldots x_{n}=r \lambda_{s 2},
$$

colla condizione $\lambda_{1}^{2}+\lambda_{2}^{2}+\cdots+\lambda^{2}=1$. Di qui si ricava

$$
d x_{1}^{2}+d x_{2}^{2}+\cdots+d x_{n}^{2}=d r^{2}+r^{2} d A^{2},
$$

dove $d \Lambda^{2}=d \lambda_{i}^{2}+d \lambda_{2}^{2}+\cdots+d \lambda_{n}^{2}$, epperi

$$
d s^{2}=\left(\frac{\operatorname{Ra}(r)}{a^{2}-r^{2}}\right)^{2}+\frac{R^{2} r^{2}}{a^{2}-r^{2}} d \mathrm{~A}^{2} .
$$

Ma chiamando $\rho$ la distanza gcodetica dall origine, o polo, al punto $\left(x_{1}, x_{2}, \ldots x_{n}\right)$, si ha

$$
\frac{R a d r^{2}}{a^{2}-r^{2}}=d_{\rho}, \quad \frac{r^{2}}{a^{2}-q^{2}}=\operatorname{senh}^{2}-\frac{\hat{l}}{l^{2}},
$$


dunque

$$
d s^{2}=d \rho^{2}+\left(R \operatorname{senh} \frac{\rho}{R}\right)^{2} d \Lambda^{2}
$$

forma che giusiivica la denominazione di polare, poichè in essa le variabili sono il raggio vettore $\rho$, e le quantità $\lambda$ che definiscono la direzione di questo raggio.

Da questa forma si passa facilmente ad un'altra che si potrebbe chiamare stereografica, e che si ottiene ponendo

$$
\xi_{r}=2 R \operatorname{tg} h \frac{p}{2 R} \cdot \lambda_{r}
$$

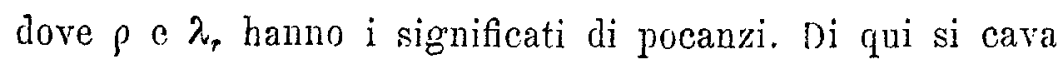

$$
\begin{aligned}
& \lambda_{r} d \rho+R \operatorname{senh} \frac{\hat{\theta}}{R} \cdot d \lambda_{r}=d \xi_{r} \cdot \cosh ^{2} \frac{\rho}{2 R}, \\
& \cosh ^{2} \frac{i}{2 h}=\frac{1}{1-\frac{c_{1}^{2}+\frac{2}{2}+\cdots+c^{2}}{4 h^{2}}},
\end{aligned}
$$

e quindi, quadrando e sommando le equazioni che risultano dalla penultima col farc $r=1,2, \ldots n$, con riguardo all' ultima cd alla $(18)$,

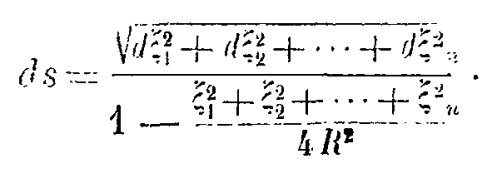

Questa forma ì stata indicata senza dimostrazione da RrFusxi, nolla citata Nemoria post:uma (II, §4).

Riemaxis ha indicato un altro sistema di coordinate, dal quale egli trae la misura delle curvature di un dato spazio intorno ad un punto (II, 8 2). Qucste coordinate sono per certi rispetti analoghe alle ortogonali cartesiane, poiche si ottcrigono dalle polari col porre

$$
z_{1}=\rho \lambda_{1}, \quad z_{2}=\rho \lambda_{2}, \ldots, z_{n}=\rho \lambda_{n} \text {. }
$$

Da queste si ha

$$
d \lambda_{s}=\frac{\hat{\theta} d z-z d s}{i^{2}}
$$


epperó, quadrando e sommando,

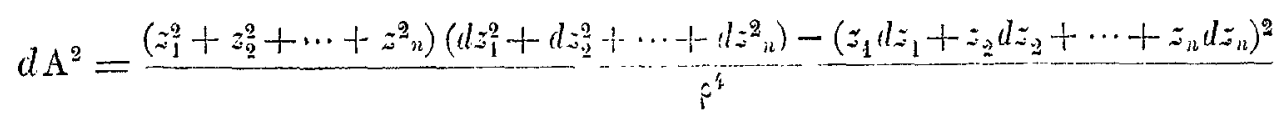

ossia

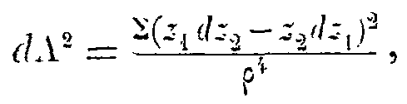

dove il segno $\$$ comprende tutte lo combinazioni binario degli indici. lia pure

$$
d \rho^{2}=d z_{1}^{2}+d z_{z}^{2}+\cdots+d z_{n}^{2}-\frac{\Sigma\left(z_{1} d z_{2}-z_{2} d z_{1}\right)^{2}}{\beta^{2}},
$$

laoude, sostituendo nella (18), si ottieno finalmente

$d s^{2}=d z_{1}^{2}+d z_{2}^{2}+\cdots+d z_{n}^{2}+\frac{1}{p^{2}}\left\{\left(\frac{R}{p} \operatorname{sen} h_{1}-\frac{p}{R}\right)^{2}-1\right\} \mathrm{y}\left(z_{1} d z_{2}-z_{2} d z_{1}\right)^{2},(20)$

ossia

$d s^{2}=d z_{1}^{2}+d z_{3}^{2}+\cdots+d z^{2}+\frac{1}{3 n^{2}}\left(1+\frac{2 ?^{2}}{13 !^{2}}+\cdots\right) y^{2}\left(z_{1} d z_{2}-z_{2} l z_{1}\right)^{2}$

dove $\rho^{2}=z_{1}^{2}+z_{2}^{2}+\cdots z_{n}^{2}$, e dove la seric convergente posta fra parentesi procede secondo le potenze crescenti di $\frac{\rho}{R}$. Per piccolissimi valori di $\rho$ si puó prendere semplicemente

$$
d s^{2}=d z_{1}^{2}+d z_{2}^{2}+\cdots+d z^{2}{ }_{n}+\frac{1}{3 n^{2}} z^{2}\left(z_{1} d z_{2}-z_{2} d z_{1}\right)^{2} .
$$

Ora considerando un elemento di superficie passante per l'origine, si può fare in modo (con un'opportuna scelta degli assi $z_{1}, z_{2}, \ldots$, ossia $x_{1}, x_{2} \ldots$ ) che esso coincida con quello della superficie $z_{3}=0, z_{4}=0, \ldots z_{n}=0$, alla quale corrisponde, nelle vicinanze dell'origine, l'elemento lineare

$$
d s^{2}=d z_{1}^{2}+d z_{2}^{2}+\frac{1}{3 R^{2}}\left(z_{1} d z_{2}-z_{2} d z_{1}\right)^{2}
$$

e poichè l'area del triangolo infinitesimo che ha $i$ vertici nei punti $(0,0)$, $\left(z_{1}, z_{2}\right),\left(d z_{1}, d z_{2}\right)$, dei quali il secondo $\dot{e}$ infinitamente vicino all'origine, $\dot{\partial}=\frac{1}{2}\left(z_{1} d z_{2}-z_{2} d z_{1}\right)$, se ne conclude che $\Sigma\left(z_{1} d z_{2}-z_{2} d z_{1}\right)^{2}$ ì eguale al quadruplo del quadrato dell'area del triangolo infinitesimo che ha i vertici nei punti $(0,0, \ldots 0),\left(z_{1}, z_{2}, \ldots z_{n}\right),\left(d z_{1}, d z_{2}, \ldots d z_{n}\right)$ il secondo dei quali $\grave{c}$ 
infinitamente vicino all'origine. Se dunque si divide la somma dei termini di $4^{\circ}$ ordine nella $(20)^{\prime}$ per il quadrato dell' area del triangolo infinitesimo anzidetto, si ha il quoziente $\frac{4}{3 R^{2}}$; e poichè, secondo la definizione di RiEuans, tale quoziente moltiplicato per - 5 esprime la misura della curvatura nel senso dell'elemento superficiale anzidetto, si vede che nello spazio qui considerato tale misura è costante ed $=-\frac{1}{R^{2}}$ in ogni direzione intorno a ciascun punto $\left(^{*}\right)$. Egli è percio che questo spazio puỏ acconciamente esser chiamato di curvatura costante.

Una quarta transformazione, importantissima, è quella che si ottiene introaucendo $n$ nuove variabili indipendenti $n, n_{1}, \ldots n_{n-1}$ e ponendo

$$
\frac{R x}{a-x_{n}}=n, \quad \frac{R x_{1}}{a-x_{n}}=n_{1}, \ldots \quad \frac{R x_{n-1}}{k-x_{n}}=n_{n-1} .
$$

Se ne trae immediatamente

$$
d s=R \frac{\sqrt{d r_{1}^{2}+d r_{1}^{2}+\cdots+d r_{1}^{2} n-1}}{x^{2}}
$$

(*) Per vedere la coincilunza della definiziono di Rifmans con quella di Gauss, si rammenti che, secondo Gauss, la misura della curvatura della superficie definita dall'elemento

$$
d s^{2}=d s^{2}+n^{2} d g^{2}
$$

i espressa da $-\frac{1}{m} \frac{\partial^{2} m}{\partial \hat{p}^{2}}, m$ essendo funzione in generale di $\hat{i}$ e di $\theta$. Se la variabile $p$ è la lunghezza di un arco geodetico uscente da un punto della superficio nel quale questa abbia una curvatura ordinaria, la funzione $m$ è della forma $m=\hat{p}\left(1+m^{\prime} \hat{\imath}^{2}\right)$ dove $m^{\prime} \dot{e}$ una funzione che per $\rho=0$ non è nè nulla né infinita (veggansi p. cs. questi Annali, p. 3038 dol tomo prec.) o quindi la misura della curvatura nel punto $\hat{\imath}=0 \dot{b}=-6 m_{0}^{\prime}$. Ciò posto, le coordinate di RHEMANN

$$
z_{1}=p \cos \theta, \quad z_{2}=0 \operatorname{sen} \theta
$$

danno all'elemenlo testè considerato la forma

$$
d s^{2}=d z_{1}^{2}+d z_{2}^{2}+\frac{4\left(m^{2}-\rho^{2}\right)}{\rho^{4}}\left(\frac{z_{1} d z_{2}-z_{2} d z_{1}}{2}\right)^{2},
$$

cpperò la misura della curratura nel punto $q=0$ é, secondo Riexss.s, $-\frac{3}{4} \lim \frac{4\left(m^{2}-p^{2}\right)}{p^{4}}$. Ora $\lim \frac{m^{2}-p^{2}}{\rho^{4}}($ per $p=0)=2 m_{0}^{\prime}$; dunque le due espressioni coincidono.

is chiaro clse $m_{0}^{\prime}$ cioè $\left(m^{\prime}\right)_{\rho=0}$ dev'essere una quantità indipendente da $\theta$. 
donde si conclude intanto che la formola (1) rappresenta l'clemento lineare di uno spazio di curvatura costante anche quando le $n+1$ rariabili $x, x_{1}, \ldots x_{n}$ sono indipendenti fra loro e non punto legate dalla relazione (2), salvo che in questo caso il numero delle dimensioni dello spazio ì $n+1$ e non sussiste più la proprietà che le linee geodetiche sono rappresentate da equazioni lineari (*). Ma una conseguenza assai notabile che si deduce dalla espressione (21) è che lo spazio ad $n-1$ dimensioni $n=$ cost. ha la sua curvatura nulla in ogni punto, poichè il suo elemento lineare la la forma

$$
d s=\text { cost. } \sqrt{d} \overline{\eta_{1}^{2}+d x_{12}^{2}+\cdots+d \eta_{n-1}^{2}} .
$$

Ed infatti, se si pon mente alla formola di Rremann (19) si vedo subito che l'elemento non può ridursi ad essere la radice quadrata della somma dei quadrati di tanti differenziali esatti quante sono le dimensioni, se non si abbia $\frac{1}{R}=0$. Lo spazio $\eta=$ cost. 0 dunque uno di quelli che Rremsan denomina piani (II, §1) e nei quali rientrano il piano o lo slazio ordinario, definiti dalle formole

$$
d s=\sqrt{d x^{2}+d y^{2}}, \quad d s=V d x^{2}+d y^{2}+d z^{2} .
$$

Ora l'equazione $\eta=$ cost. ammette una molto semplice interpretazione, dietro quanto precede. Il punto all'infinito sull' asse delle $x_{n}$ ha per coordinate

$$
x_{1}=x_{2}=\cdots=x_{n-1}=0, \quad x_{n}=a,
$$

e quindi l'equazione (13) diventa per esso

$$
\frac{a-x_{n}}{x}=k^{\prime} e^{-\frac{\varphi}{n}}
$$

dore $k^{\prime}=\frac{k}{a} \cdot$ Dunque

$$
y=\frac{R}{l^{\prime}} e^{\frac{p}{h}}
$$

epperò l'equazione $\eta=$ cost. equivale a quest' altra $\rho=$ cost., donde si conclude (poichè è arbitraria la direzione dell'asse delle $x_{n}$ ) che lo spazio ad $n-1$ dimensioni $\eta=$ cost. non è altro che una delle trajettorie ortogonali

(*) La forma (21) i stata indicata, pel' il caso di duo sole dimensioni, dal sig. Liouvuce, nelle sue nolo all'opera di Mosie, 1'.600. 
di tutte le geodetiche convergenti verso uno stesso punto all'infinito, cioè di un sistema di geodetiche parallele fra loro. Reciprocamente ciascuma di queste trajettorie ortogonali ha in ogni punto la curvatura nulla, epperó due qualunque di esse (appartenenti o meno al medesimo sistema) sono sovrapponibili l'una all'altra in tutti i modi possibili.

Introducendo nella (21) la variabile $\rho$ al posto della $\eta$ si ha l'aitra forma equivalente

$$
d s^{2}=d \rho^{2}+k^{2} e^{-\frac{2 o}{k}}\left(d x_{1}^{2}+d \eta_{2}^{2}+\cdots+d \eta^{2}{ }_{n-1}\right) .
$$

si è già veduto che il complesso di $n-1$ equazioni lineari fra le coordinate $x_{1}, x_{2}, \ldots x_{n}$ rappresenta una linea geodetica. Vediamo cosa rappresenti, più in generale, il complesso di $n-m$ equazioni lineari.

Supponendo dedotte da queste equazioni le espressioni di $n-m$ coordinate in funzione delle rimanenti $m$, riesce manifesto che il numero dei parametri indipendenti contenuti in un tal sistema e $(m+1)(n-m)$. Si immagini ora che tutte le $n$ coordinate $x_{1}, x_{2}, \ldots x_{n}$ vengano espresse linearmente in funzione di $m$ variabili $u_{1}, u_{2}, \ldots u_{n_{i}}$. Queste espressioni comprendono fra tutte $(m+1) n$ parametri, ma se si assogrgettano questi parametri a verificare l'identità

$$
x_{1}^{2}+x_{1}^{2}+\cdots+x_{n}^{2}=u_{1}^{2}+u_{2}^{2}+\cdots+u_{n}^{2}+l^{2}
$$

(h restando indeterminataj, è chiaro cho si aggiungrono con cio $\frac{m(m+1)}{\mathbf{z}}+m$ condizioni, talchè il numero dei parametri indipendenti rimane di $(n+1) n-\frac{m(m+1)}{2}-m$. Ora questo numero eccede di $\frac{m(m-1)}{2}$ il numero $(m+1)(n-m)$; dunque lo relazioni ammesse fra le $x$ e le $u$, colla indicata condizione, sono tali da poter sempre tener luogo, senza restrizione alcuua, del dato sistema di $n-m$ equazioni. Ciò posto, da quelle relazioni, ponendo

$$
u^{2}+u_{1}^{2}+\cdots+u^{2}=a^{2}-h^{2}=a^{2}
$$

si leduce

$$
\begin{aligned}
d x^{2}+d x_{1}^{2}+\cdots+d x_{n}^{2} & =d u^{2}+d u_{1}^{2}+\cdots+d u^{2}{ }_{m}, \\
x^{2} & =u^{2},
\end{aligned}
$$

dunque:

$$
d s=R \frac{\sqrt{d u^{2}+d u_{1}^{2}+\cdots+d u_{n}^{2}}}{u}
$$


colla condizione

$$
u^{2}+u_{1}^{2}+\cdots+u^{2}{ }_{n i}=u^{i^{2}} .
$$

Conseguentemente il luogo dei punti rappresentati dal complesso delle $n-m$ equazioni lineari fra le coordinate $x_{1}, x_{2}, \ldots x_{n}$ ¿ uno spazio ad $m$ dimensioni, la cui curvatura ò dovunque costante ed eguale a quella dello spazio primitiro.

Così per es. $n-2$ equazioni lineari rappresentano una superficie di curvatura costante $\left(=-\frac{1}{R^{2}}\right)$, che conviene distinguere col nome di sujerficie di prim'ordine; $n-3$ rappresentano uno spazio a tre dimensioni di curvatura costante $\left(=-\frac{1}{R^{2}}\right)$; ecc.

Una linea georletica reale ì individuata scnza ambiguita da due punti dello spazio: nelle ipotesi fin qui ammessc non è possibile alcuna eccezione a questa proprietà.

Una superficie di prim' ordine è individuata senza ambiguità da tre punti dello spazio. Essa contiene tutta intera la geodetica che passa per due suoi punti reali, talchò se due superficie reali di prim'ordine hanno due punti reali in comune, hanno del pari in comune tutta la geodetica individuata da questi.

Un triangolo geodetico griace sempre sopra una determinata superficie di prim'ordine, la quale è individuata anclio quando il triangolo è infinitesimo. Perció se si prolungano sccondo linee geodetiche tutti gli elementi lineari contenuti in uno stesso elemento di supericie, le linee geodetiche cosi ottenutc hanno tutte un luogo geometrico che è una determinata superficie di prim'ordine.

Quando due superficie di prim' ordine si intersecano lungo una linea, necessariamente geodetica, il loro angolo è dovunque costante; ciò̀ condotti da un punto della loro intersezione due elementi lineari normali ad essa, l'uno nella prima, l'altro nella seconda superficie, la distanza infinitesima dei loro termini è costante, se sono costanti le loro lunghezre. Infatti (*) supposto diretto l'asse $x_{1}$ secondo la comune sezione delle due superficie, le equazioni di queste possono evidentemente esser messe sotto la forma

$$
\begin{aligned}
& \left(x_{2}=m_{2} x_{n}, \quad x_{3}=m_{3} x_{n} \ldots \quad x_{n-1}=m_{n-1} x_{n}\right), \\
& \left(x_{2}=m_{2}^{\prime} x_{n}, \quad x_{3}=m_{3}^{\prime} x_{n}, \ldots \quad x_{n-1}=m_{n-1}^{\prime} x_{n}\right),
\end{aligned}
$$

(1) Isa saghente dimosthazionn, che poteva a rigore cssere onessa, si i inserita in grazia delle formole a cui contluce. 
dove le $m, m^{\prime}$ sono parametri costanti. Queste due superficie sono intersecate dallo spazio $x_{1}=a_{1}$ secondo duc geodetiche che, per una precedente osservazione, sono ortogonali all'asse $x_{1}$. I due punti di coordinate

$$
\begin{aligned}
& \left(x_{2}=a_{1}, \quad x_{2}=m_{2} x_{n}, \ldots \quad x_{n-1}=m_{n-1} x_{n}, \quad x_{n}=x_{n}\right), \\
& \left(x_{2}=a_{1}, \quad x_{2}=m_{2}^{\prime} x^{\prime}{ }_{n}, \ldots \quad x_{n-1}=m_{n-1}^{\prime} x_{n}^{\prime}, \quad x_{n}=x_{n}^{\prime}\right) \text {, }
\end{aligned}
$$

giacciono rispettivamente sulla prima e sulla seconda superficie, e precisamente sulle due geodetiche anzidette, e la loro distanza $\rho$ è data (8) dalla formola

$$
\cosh \frac{P}{R}=\frac{a^{2}-a_{1}^{2}-M x_{n} x_{n}^{\prime}}{\sqrt{\left(a^{2}-a_{1}^{2}-m^{2} n^{2}\right)\left(n^{2}-a_{1}^{2}-m^{1^{2}} x_{n}^{t^{2}}\right)}},
$$

dore si è posto

$$
\begin{gathered}
m^{2}=1+m_{2}^{2}+\cdots+m_{n-1}^{2}, \quad m^{\prime 2}=1+m_{2}^{\prime 2}+\cdots+m_{n-1}^{\prime^{2}}, \\
M=1+m_{2} m_{2}^{\prime}+\cdots++m_{n-1} m_{n-1}^{\prime} .
\end{gathered}
$$

Da essa, chiamando $\sigma, \sigma^{\prime}$ le lmighezze delle due geodetiche comprese fra il punto comune $x_{1}=a_{1}$ ed i due punti considerati, si trae

$$
\cosh \frac{\sigma}{R}=\frac{\sqrt{a^{2}-a_{1}^{2}}}{\sqrt{a^{2}-a_{1}^{2}-m^{2} x^{2}{ }_{a}^{2}}}, \quad \cosh \frac{\sigma^{\prime}}{R}=\frac{\sqrt{a^{2}-a_{1}^{2}}}{\sqrt{a^{2}-a_{1}^{2}-m^{\prime^{2} x_{n}{ }^{a^{2}}}}},
$$

e quindi

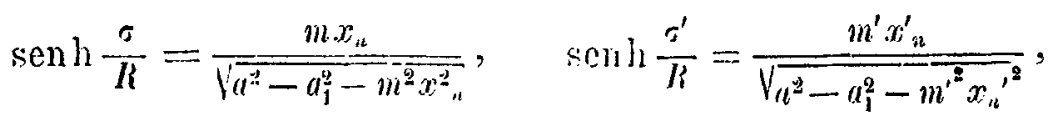

valori i quali mostrano che

$$
\cosh \frac{\theta}{R}=\cosh \frac{\sigma}{R} \cosh \frac{\sigma^{\prime}}{R}-\frac{M}{m m^{\prime}} \operatorname{senh} \frac{\sigma}{R} \operatorname{senh} \frac{\sigma^{\prime}}{R} \text {. }
$$

Siccome in questa formola non resta piu traccia del punto $a_{1}$ preso sull'asse $x_{1}$, cosi si rede che da qualunque punto di questo asse si conducano nelle d.ue superficie le geodetiche di lunghezza $\sigma, \sigma^{\prime}$, la distanza geodetica dei loro estremi $\mathrm{i}$ sempre costante. E poichè questa proprictà sussiste per Iunghezze $\sigma, \sigma^{\prime}$ qualunque, necessariamente sussiste per lunghezze infinitesime, donde scaturisce il tevrema annunciato.

Ammettendo, come gia si $i$ fatto implicitamente nel porre la condizione 
di ortogonalità, che i triangoli inf̂nitosimi siano soggretti allo relazioni della ordinaria trigonometria piana, si riconosce immediatamente, reudendo infinitesime lo lungherzo $\rho, \sigma, \sigma^{\prime}$, che $\frac{M I}{m m^{\prime}}$ i il coseno dell angolo hat to thi prini elementi delle due geoktiche $\sigma, \sigma^{\prime}$, cios delle duo supericic. D'altra parte ¿ facile vedere che il triangolo ora considerato puo esscre un triangolo geodetico interamente arbitrario; dunque fra i lati $a, b, c$ e gli angoli opposti $A, B, C$ di un triangolo greodetico esistente nello spazio cousiderato, sussiste la relaziono

$$
\cosh \frac{a}{d t}=\cosh \frac{b}{R} \cosh \frac{b}{h}-\operatorname{senh} \frac{b}{l n} \sinh \frac{c}{R} \cos 1
$$

insieme colle sue analoghe, bi quaic non difrerisee dalla formola fondamentale della trigonometria sferica che per il cambiamonto di $R$ in $R V-T$ ( $R$ ragroio della sfera), rimanendo invariati i lati o gli angoli. Cio concorda pienamonto con un fatto già avrertito dal MrNorke (nel t. XX del Giomale di Cnerue) c dimostrato dal Codszzi (negli Annali di 'Tor'rocine, 18.57), se si rammenta che il triangolo geodotico qui considcraro giace intieramente sopra una superficic di prim'ordine, cioè di curvatura costante negativa, rispetto alla quale csso è pure gondetico nel scnso ordinario. Se si suppone retto l'angolo $G$, le due formole che si deducono dalla (22) colla permutazione degli elementi danno, opportunamente combinate.

$$
\operatorname{tg} h \frac{a}{l l}=\operatorname{tg} h \frac{c}{R} \cos l \text {. }
$$

Se ora si imagian che il vertice dellangolo 4 vada indefintamente allontanandosi sul catcto $b$, mentro il lato a rinane invariato di posizione e di grandezza, l'ipotenusa $c$ crescerà. fino all'infinito, ed a questo limite le equazioni (22) (23) daranno

$$
\cos A=1, \quad \operatorname{tgh} \frac{a}{R}=\cos B .
$$

La prima folmola insegna cho $A=0$, ciod che i duc lati $b, c$ si accostano assintoticamentc, quando il vertice dell' angolo $A$ c all'infinito; la seconda che il limito dell' angolo $B$ non è l'angolo retto, come nel piano, ma un angolo minore di $y^{\circ}$, la cui grrandezza dipende dalla distanza $a$, mediante la formola

$$
\lg \frac{b}{2}=a^{-\frac{n}{1 i}}
$$


(equivalente alla superiore). Se si chiamano parallele due geodetiche con. vergenti verso un medesimo punto all'infinito, come già si è fatto, si vede dunque che da un punto si possono condurre due distinte geodetiche parallele ad una geodetica data, che queste due parallele sono egualmente inclinate da una farte e dall' altra della geodetica condotta dallo stesso punto normalmente alla data, e che la loro inclinazione $B$ sulla normale è legata alla lunghezza $a$ di questa stessa normale mediante la relazione (24). Questo risultato s'accorda pienamente con quello che forma la base fondamentale della geometria non-euclidea, i cui principii, già famigliari a Gauss, sono stati compendiati maestrevolmente da Lobatschewsiry nella Théorie des paralliles (trad. HoüEr), sotto una veste sintetica. La possibilità della sua costruzione col mezzo dell'ordinaria sintesi (limitatamente allo spazio di tre dimensioni) dipendo in primo lungo da ciò che, come si è dimostrato, negli spazii di curvatura costante (positiva o negativa) ogni figura $\mathrm{pud}$ ussere mutata arbitrariamente di posizione senza subire alcuna alterazione nella grandezza e nella disposizionc mutua dei suoi elcmenti contigui, possibilità da cui dipende l'esistenza delle figure eguali e quindi la validita del principio di sorrapposizione. In secondo luogo negli spazii di curvatura costante negativa le geodetiche sono caratterizzate, come la retta euclidea, dalla proprieta di essere individuate senza ambiguità da due soli dei loro punti, talchè vige per esso l'assioma della retta. E del pari le superficie di prim'ordine sono caratterizzate, come il piano cuclideo, dalla propricta di esscre individuate senza ambiguita da tre soli dei loro punti, talchè rige per esse l'assioma del piano. Inoltre le relazioni delle linee gcodeticlic colle superficie di prim'ordine e di queste fra loro, sono le stesse di quello dello rette coi piani e dei piani fra loro, poiche una di quelle superficie contiene tutta una greodetica tosto che ne contiene due punti, e due di quelle superficie si scgano secondo una gendetica (c sotto un angolo costante) se s'incontrano in un solo punto. Da questa corrispondenza consegrue cho se si ammettono grli assiomi fondamentali della geometria ordinaria, escludendo il postulato dollo parallele, i teoromi che si ottengono sono eguali a quelli della geometria dello spazio di curvatura costante negativa, poichè questa secunria geometria lia le stesse basi di quella, trame il postulato auzidetto. I teoremi di essa sussistono por orna valore della curratura, che $i$ il parametro della geomitria non-enchica (la quale io propongo di ienominare pscudosferica), o le sole misure prese nello spazio obhicttiro possono far riconoscere che il valore speciale 
della sua curratura è $z$ ero, ciò̀ che $R=\infty$ pur esso; nello stesso modo che per sole misure si può assognare la curvatura di una sfera data, che e il parametro della geometria sferica.

Efrettivamente si puo rerificare che la teoria di Lobatscrumsir coincide, salro nei nomi, colla geometria dello spazio a tre dimensioni di curvatura costante negativa. Chi ami vedere sviluppata questa corrispondenza ne potrà trovare altrove una più minuta esposizione (*). Qui, per non fare una troppo lunga digressione, mi limiterò ad alcuni cemni sommarii.

La planimetria non-euclidea non é altro che la geometria delle superficie di curvatura costante negativa. Lo circonferenzo di quella corrispondono alle linco clic tagliano ortogonalmente tutti i ragoi greodetici uscenti da uno stesso punto della superficie, ossia alle circonferenze geodetiche. Il perimetro ne $\dot{c}$ dato in funziono del ragrgio geodetico $r$ dalla bornola

$$
\pi R\left(e^{\frac{i}{h}}-e^{-\frac{r}{h}}\right)
$$

come aveva già enunciato Gauss. Por tro punti della superficie non si può scmpre far passarc una circonferenza geodetica avente il centro in un punto reale. Gli oricicli o curve-limiti ai Lobatscinwsiry non sono altro cho le circonferenze geodetiche il cui contro è all'infinito, cioc̀ i cui raggi formano un sistema di geodetiche parallele. Facendo nella $(21)^{\prime} n=2$ si ha

$$
d s^{2}=d \rho^{2}+k^{2} e^{-2} i^{-2} d n^{2},
$$

espressione dell' elemento linearo della supcricie di curvatura costante negativa riferita ad un sistema di oricicli concentrici ed ai loro raggi. La forma di quest'espressione insegna che gli oricicli possono diventare, mercè una flessione opportuna della superficic, i paralleli dolla superficie di rotazione il cui meridiano ì la curva delle tangenti di lungliczza costante $=R$.

La stereometria non-euclidea non è altro che la geometria degli spazii a tre dimensioni di curvatura costante negativa. Si è già detto a che corrispondano, in questa geometria, lo rette ed i piani. Alle superficie sferiche corrispondono le superficie che tagliano ortogonalmente tutti $i$ raggi geodetici uscenti da uno stesso punto, ciò le sfere geodetiche. Anche qui può

(*) Si rorra il Giolntlo Matematico di Napoli, settembre-oltobre 1868 , dove le particolarita srolte per il caso di due dimensioni si possono agevolmente ripolore per quello di the, massime se si tien conto doi risultati dol presente suritto e se si ricorro ald una sfora ausiliare. 
darsi che per tre punti, e molto piủ per quattro, non si possa far passare una sfera geodetica col centro in un punto reale. Le orisfere o superficie-limiti di Lonatschewsky (*) non sono altro che le sfere geodetiche il cui centro è all'infinito, ciò̀ i cui raggi formano un sistema di geodetiche parallole dello spazio di curvatura costante negativa. Facendo nella (21) $n=-3$ si ha

$$
d s=R \frac{\sqrt{\left(r_{1}^{2}+d r_{1}^{2}+d r_{1}^{2}\right.}}{r_{1}}
$$

dove

$$
\frac{R x}{a-x_{3}}=n, \quad \frac{n x_{1}}{a-x_{3}}=n_{1}, \quad \frac{R x_{2}}{a-x_{3}}=n_{2},
$$

e reciprocamento

$$
\begin{gathered}
x_{1}=\frac{2 a R r_{1}}{r_{1}^{2}+r_{1}^{2}+r_{12}^{2}+n^{2}}, \quad x_{2}=\frac{2 a R r_{2}}{r_{1}^{2}+r_{1}^{2}+r_{2}^{2}+n^{2}} \\
x_{3}=\frac{a\left(r_{1}^{2}+r_{1}^{2}+r_{12}^{2}-R^{2}\right)}{r_{1}^{2}+r_{1}^{2}+r_{12}^{2}+h^{2}} .
\end{gathered}
$$

La furmola (25) rappreserta l'elemento lineare dello spazio non-euclideo riferito ad un sistema di orisfere concentriche ed a quello dei loro raggi. La forma di questo elemento insegna che ogrii orisfera, essendo rappresentata da $n=$ cost., è una superficie di curvatura nulla, poichè il suo elemonto lineare ha la forma

$$
d s=\text { cost. } \sqrt{d \eta_{1}^{2}+d \eta_{2}^{2}}
$$

e che le variabili $\hat{n}_{x}, \eta_{2}$ sono le coordinato rettangole do suoi punti. Una superficie di prim'ordine

$$
l x_{1}+m x_{2}+n x_{3}+p=0
$$

c rappresentata in coordinate $\eta_{1} \eta_{1}: \eta_{2}$ dall' equaziono

$$
2 a R\left(l n_{1}+m n_{2}\right)+(a n+p)\left(n^{2}+n_{1}^{2}+n_{2}^{2}\right)=(a n-p) R^{2},
$$

epperò taglia l'orisfera (per la quale $\eta=$ cost.) secondo un cerchio. Questo si ridnce ad ma retta solamente quanlo $p=-a n$, cios quando l'equaziono

() Ossia le superlieir $F^{*}$ di l, Butrat. 
delia superficie di prim' ordine ha la forma

$$
l x_{1}+m x_{2}+n\left(x_{3}-a\right)=0
$$

il che accade quand'essa è una superficie diametrale dell' orisfera, ossia passa pel centro (all'infinito) di questa. In questo caso la linea d'intersezione è evidentemente un oriciclo di questa superficic diametrale, mentre rispetto all' orisfera $\dot{e}$ tale che si converte in una retta quando questa venga distesa secondo un piano. Di qui emerge che il triangolo tracciato sopria un'orisfera da tre superficie diametrali $\grave{e}$ in sostanza un triangolo greodetico esistente in una superheio li curratura nulla, il quale percio soddisfa a tutte le relazioni dell' ordinaria trigonometria piana, poichè es esattamente applicabile sopra un triangolo rettilineo.

Cosi tutti i concetti della geometria non-euclidea trovano un perfetto riscontro nella geometria dello spazio di curvatura costante negativa. Solamente fa d'uopo osservare che mentre quelli relativi alla semplice planimetria ricevono in tal modo un' interpretazione vera e propria, poiche diventano costruibili sopra una superficic reale, 'uelli all'incontro che abbracciano tre dimensioni non sono suscettibili che di una rappresentazione analitica, poichè lo spazio in cui talo rappresentazione vcrrebbe a concretarsi è diverso da quello cui generalmento diamo tal nome. Per lo meno l'esperienza non sembra poter essere messa d'accordo coi risultati di questa geometria più generale, se non si suppone infinitamente grande la costante $R$, cioè nulla la curvatura dello spazio; il che per altro potrebbo non essere dovuto che alla piccolezza dei triangoli che noi possiamo misurare, ossia alla piccola estcusione dello spazio a cui le nostre osservazioni si estendono, non altrimenti da ciò che accade per lo misuro prese sopra una piccola parto di superficie terrestre, la precisione delle quali non $\dot{c}$ sufficiente a mettere in evidonza la sfericità dol globo.

Tin qui non si è parlato che di spazii ad $n$ dimensioni la cui curvatura i) costante, ma negativa; dol che d causa l'aversi avuto principalmente in vista il ravvicinamento dei concetti ad essi relativi con quelli della geometria noli-euclidea, rispetto alla quale l'ipotesi opposta ha minore interesse. Nondimeno so no diranno qui alcune poche cose.

L' elemonto lincare

$$
d s=R \frac{\sqrt{a} x_{1}^{2}+d x_{2}^{2}+\cdots+d x^{2}-a x^{2}}{x}
$$


dove

$$
x^{\prime \prime}=u^{2}+x_{1}^{2}+x_{a}^{2}+\cdots+x^{2}{ }_{n},
$$

appartiene ad uno spazio di $n$ dimensioni la cui curvatura è dorunque costante ed $=\frac{1}{n^{2}}$. Esso si ottiene da (1) mutando $R, a$ ed $x$ in $R \sqrt{-1}, a \sqrt{-1}, x \sqrt{-1}$, c tuttc le proprietà e le equazioni fondate sopra mere trasformazioni analitiche dell'elemento (1) valgono evidentemente, coi cambiamenti indicati, anche per quest'altro. Per es. la (8) si muta nella seguente

$$
\cos \frac{p}{R}=\frac{a^{2}+x_{1} x_{1}^{n}+x_{2} x_{2}^{n}-\cdots+x_{n} x_{n}^{n}}{\sqrt{\left(a^{n}+x_{1}^{2}+\cdots+x_{n}^{2}\right)\left(l^{2}+x_{1}^{0^{2}}+\cdots+x_{n}{ }^{2}\right)}},
$$

formola che dì per $p$ un valore reale, qualunque siano $i$ valori reali di $x_{1}, x_{2}, \ldots x_{n} ; x_{1}^{0}, x_{3}^{n} \ldots x_{n}^{n}$. È chiaro che per questi spazii sussiste integralmonte il teorema della sovrapponibilità di due loro porzioni qualunque.

Se nella (20) si suppongono reali lo variabili $x, x_{1}, \ldots x_{n}$ e le costanti $R, a$, i valori ammissibili per le coordinate $x_{1}, x_{2}, \ldots x_{n}$ non hanno limite alcuno, c possono variare fra $-\infty \mathrm{e}+\infty$. Per tutti i valori reali di queste coordinate lo spazio e continuo e semplicemente connesso, ma non infinito (Resux, III, \$2), perchie se si fa nella (27)

$$
x_{1}^{n}=i_{1} \tau, \quad x_{2}^{0}=i_{2} \tau, \ldots \quad x^{0}{ }_{1}=\lambda_{\tau} \tau
$$

dove $\lambda_{i}^{2}+2 z_{i}^{2}+\cdots+\lambda_{n}^{2}=1$, si ha por $x=y$

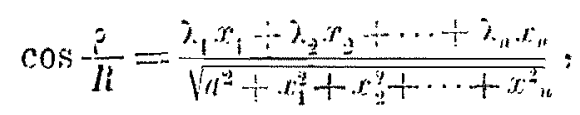

formola che da per $\rho$ un valore finito e determinato. Le linec geodotiche continuano al essere rappresentate da equazioni lineari, ma, stante l'ammissibilita dei valori infiniti per lo coordinate, il principio che due punti individuano scnza ambiguità una geodetica cessa d'esser vero senza restrizione. Infatti siano

$$
x_{1}=b_{1} x_{n}+b_{1}^{\prime}, \quad x_{2}=b_{2} r_{n}+b_{2}^{\prime}, \ldots \quad \text { ccc. }
$$

le cquazioni d'una geodetica. Finchie uno almono dei punti pei quali essa dere passare ha le sue coorlinate finite, i conficienti possono esser tutti doterminati scnza ambiguiti. Ma so ambeduc i punti hanno coordinate infi- 
nite bisogna mettere le equazioni sotto la forma

$$
\frac{x_{1}}{x_{1}}=b_{1}+\frac{b_{1}^{\prime}}{x_{12}}, \quad \frac{x_{2}}{x_{13}}=b_{2}+\frac{b_{2}^{\prime}}{x_{13}}, \cdots \quad \text { ecc. }
$$

e sostituire ai primi membri i valori limiti a cui convergono nei due punti. Se questi limiti sono eguali in entrambi, i valori dei secondi coefficienti restano indeterminati e la linea geodetica non è più unica ed individuata. Se poi i limiti sono diversi lo coordinate della linea geodetica sono infinite in ogni punto.

Le considerazioni che hanno condotto all'equazione (13) non sono applicabili agli spazii di curvatura costante positiva, poiche non existono, per questi, punti all'infinito. Quindi gli enti rappresentati da quella cquazione non hanno riscontro in questi nuovi spazii, como non lo hamno le geodetiche reciprocamente parallole.

Si vede che la geometria degli spazii di curvatura costante positiva (che può acconciamente esser cliamata geometria sforica in senso largo, stantechè, come insegna l' equazione (22), i triangoli goodetici vi soggiacciono allo leggi della trigonometria sferica), diferisce molto notabilmente dalla pseud osferica, sebbene abbia con questa in comme l'esistenza dolle figure cguali. Del resto la gromotria psendosferica conduce spontancamente a considerare gli spazii di curvatua costinte positiva. Infatti ponendo nella (20)

$$
\frac{a}{x}=y, \quad \frac{x_{1}}{x}=y_{1}, \ldots, \frac{x_{n}}{x}=y_{i},
$$

si trova

colla condizione

$$
d s=11 \sqrt{1 y^{2}+d y_{1}^{*}+\cdots+d y^{2}}
$$

$$
y^{2}+y_{1}^{2}+\cdots+y^{2}=1
$$

risultato il quale, posto a riscontro colla equazione (18) in cui siasi fatto $\rho=$ cost. insegna che le sfere geodetiche di raggio $\rho$ nello spazio ad $n$ dimensioni di curvatura costante negativa $-\frac{1}{k^{2}}$ sono spazii ad $n-1$ dimensioni di curvatura costante positiva $\left(\frac{1}{R \operatorname{sen} h \frac{P}{R}}\right)^{2}$. Quindi la geometria sferica puo risguardarsi come contenuta nella psoulosferica.

Bologna, agosto 1868. 